Kippenberger: Zur maassanalytischen Bestimmung der Alkaloide, 101

Zur maassanalytischen Bestimmung der Alkaloide.

Yon

\title{
Prof. Dr. C. Kippenberger.
}

In den Berichten der deutschen chemischen Gesellschaft zu Berliu 1899 (32) S. 2871 veröffentlichte H. M. Gordin zwei neue Methoden zur Bestimmung der Alkaloide unter Anwendung der Reagentien nach Wagner (Jod-Jodkaliumlösung) und nach Mayer (Quecksilberjodid-Jodkaliumlösung) in der Annahme, dass der durch diese Reagentien. mit den in überschüssiger Säure gelösten Alkaloiden erzengte Niederschlag die Zusammensetzung (Alkaloid $\mathrm{H} J)_{\mathrm{m}} J_{\mathrm{n}}$, beziehungsweise (Alkaloid $\mathrm{HJ})_{\mathrm{m}}\left(\mathrm{Hg} \mathrm{J}_{2}\right)_{\mathrm{n}}$, besitze und somit die acidimetrische Bestimmungdes Filtrats die Menge an Alkaloid berechnen lasse. Es wurden für die als einsäurige Basen geltenden Alkaloide die allgemeinen Formeln Alkaloid $\mathrm{HJ} . \mathrm{J}_{\mathrm{x}}$ und Alkaloid $\mathrm{HJ} .\left(\mathrm{Hg} \mathrm{J}_{2}\right)_{\mathbf{x}}$ angenonmen und dementsprechend analytisch erhaltene Zahlen angegeben, die gegenüber den theoretisch sich ergebenden Zahlen einigermaassen übereinstimmen. Die Differenz betrug im ungünstigsten Falle $4 \%$.

Damit wird von Gordin gleichzeitig Stellung genommen gegen eine früher von mir publicirte Arbeit, in welcher ich bei Benutzung der Jodlösung den Verbrauch an wechselnden Mengen von Jodkalium nachwies. ${ }^{1}$ ) In Folge dessen war ich zu meinem Bedauern gezwungen, die Gordin'schen Methoden einer gelegentlichen Nachprüfung zu unterziehen. Diese hat ergeben, dass die Methoden von Gordin Werthe liefern, die je nach dem Jodkaliumgehalt der Lösung, aber auch je nach dem Gehalt der Alkaloidsalzlösung an Säure, sehr veränderliche analytische Zahlen darstellen. Die von Gordin erzielten, für die Methode günstigeren Resultate dürften sich auf unzureichende Ausdehnung der Versuchsreihen und auf unglückliche Zufälle in der Wahl der Versuchsbedingungen begründen.

Als Alkaloid diente Strychnin, $\mathrm{C}_{21} \mathrm{H}_{22} \mathrm{~N}_{2} \mathrm{O}_{2}$, das sich durch Elementaranalyse und titrimetrische Untersuchung als rein erwies. Es. wurden je $0,4 \mathrm{~g}$ mit $50 \mathrm{ce}$ Normal-Salzsäure zu $100 \mathrm{cc}$ gelöst und diese Lösung, wenn nicht anders bemerkt (Versuch 19), in Anwendung gezogen. Das bei den Versuchen benutzte Wasser war mehrmals über Aetzkalk destillirt; auch die angewandten Titerflüssigkeiten waren kohlen-

Diese Zeitschrift 35, 10 ff. und 422 ff. 
Kippenberger: Zur maassanalytischen Bestimmung

säurefrei, desgleichen das Jodkalium. Ebenso reagirte die benutzte Quecksilberjodid-Jodkaliumlösung — entgegen der Quecksilberchloridlösung - neutral, so dass jede durch die Art der benutzten Lösungen etwa in Berücksichtigung zu ziehende Fehlerquelle ausgeschlossen ist.

a) Versuche mit Jod-Jodkaliumlösung.

Zur Anwendung gelangten Lösungen folgender Zusammensetzung: a $100 \mathrm{cc}$ enthaltend: $5,9 \mathrm{~g} \mathrm{KJ}$ und $0,7 \mathrm{~g} \mathrm{~J}$

\begin{tabular}{|c|c|c|c|c|c|c|c|}
\hline $100 \ll$ & $\ll$ & 3,0 & $\ll$ & $\ll$ & $\ll$ & 0,7 & $\ll \ll$ \\
\hline $100 \ll$ & $\ll$ & 1,5 & $\ll$ & « & $\ll$ & 1,0 & \\
\hline $100 \ll$ & * & 0,75 & $\ll$ & $\ll$ & $\ll$ & 0,516 & $\ll$ \\
\hline
\end{tabular}

1. $30 \mathrm{cc}$ Strychninlösung mit $25 \mathrm{cc}$ J-KJ., vermischt, nach 15 Minuten filtrirt, mit Thiosulfatlösung entfärbt, verbrauchten für $45,9 \mathrm{cc}$ Filtrat: $118,6 c e^{1 / 10} \mathrm{~N}-\mathrm{K} O H$. Es waren mithin in Form des Alkaloidsalzes vorhanden: $7,9 c c 1 / 10 \mathrm{~N}-\mathrm{HCl}$. Nach Gordin berechnen sich 3,57 cc. Es wurden also gefunden: $221 \%$ Strychnin anstatt $100 \%$.

2. $10 c c$ Strychninlösung mit $25 c c \mathrm{~J}-\mathrm{KJ} \alpha$ unter 5 Minuten langer gegenseitiger Einwirkung analog 1 behandelt, ergaben für 27,4 cc Filtrat: $36,5 c c{ }^{1 / 10} \mathrm{~N}-\mathrm{K}$ OH. Verbraucht: $3,4 c c 1 / 10 \mathrm{~N}-\mathrm{HCl}$ anstatt $1,19 c c$; also gefunden $285 \%$ Strychnin.

3. $30 \mathrm{cc}$ Strychninlösung mit $25 \mathrm{cc} \mathrm{J}-\mathrm{KJ} \beta$ analog 2 behandelt. $20 c c$ Filtrat $=50,25 c c^{1 / 10} \mathrm{~N}-\mathrm{K}$ OH. Verbraucht $: 12,0 c c^{1 / 10} \mathrm{~N}-\mathrm{HCl}=$ $336 \%$ Strychnin.

4. $10 \mathrm{cc}$ Strychninlösung mit $25 \mathrm{cc} \mathrm{J}-\mathrm{KJ} \beta$ analog 2 ergab nach 11 Minuten langer Einwirkung für $27 c c$ Filtrat: $35,6 c c$ 1/10 $\mathrm{N}-\mathrm{K} \mathrm{OH}$. Verbraucht: 3,9 cc $1 / 10 \mathrm{~N}-\mathrm{HCl}=327 \%$ Strychnin.

5. 30 c $c$ Strychninlösung mit $25 c c \mathrm{~J}-\mathrm{KJ} \gamma$ analog 1 behandelt. $20 c c$ Filtrat $=52,68 c c 1 / 10 \mathrm{~N}-\mathrm{K} \mathrm{OH}$. Verbraucht $: 5,23 c c 1 / 10 \mathrm{~N}-\mathrm{HCl}=$ $146 \%$ Strychnin.

6. $10 c c$ Strychninlösung mit $25 c c \mathrm{~J}-\mathrm{KJ} \gamma$ analog 1 behandelt. $27 c c$ Filtrat $=36,1 c c^{1 / 10} \mathrm{~N}-\mathrm{K} \mathrm{OH}$. Verbraucht $: 3,2 c c^{1 / 10} \mathrm{~N}-\mathrm{HCl}=$ $268 \%$ Strychniu.

7. $30 c c$ Strychninlösung wurden mit $135 c c^{1 / 10} \mathrm{~N}-\mathrm{K} \mathrm{OH}$, dann mit $25 \mathrm{cc} \mathrm{J}-\mathrm{KJ}_{\ddot{i}}$ vermischt und die Flüssigkeit mit Wasser auf $200 \mathrm{cc}$ aufgefüllt. Der Niederschlag setzte sich sehr langsam ab; erst nach 30 Minuten langem Stehenlassen konnte klar abfiltrirt werden, doch erwies sich die Fällung des Alkaloids als unvollständig, obwohl Jodjodkaliumlösung im Ueberschusse zur Anwendung gelangte. Nach 
14 stündiger Einwirkung: $50 \mathrm{cc}$ Filtrat $=2.55 \mathrm{cc}{ }^{1 / 10} \mathrm{~N}-\mathrm{KOH}$. Verbrancht: $4,8 c c{ }^{1} / 10 \mathrm{~N}-\mathrm{HCl}=134 \%$ Strychnin.

8. $30 \mathrm{cc}$ Strychninlösung mit $25 \mathrm{cc} \mathrm{J}-\mathrm{KJ} \delta$; nach einstündiger Einwirkung: $20 c c$ Filtrat $=52,93 c c{ }^{1} / 10 \mathrm{~N}-\mathrm{K} \mathrm{OH}$. Verbraucht : $4,45 c c$ $1 / 10 \mathrm{~N}-\mathrm{HCl}=124 \%$ Strychnin.

9. $10 \mathrm{cc}$ Strychninlösung mit $25 \mathrm{cc} \mathrm{J}-\mathrm{KJ} \delta$; nach 50 minutenlanger Einwirkung: $25 c c$ Filtrat $=34,27 c c^{1 / 10} \mathrm{~N}-\mathrm{KOH}$. Verbraucht : $2,03 c c 1 / 10 \mathrm{~N}-\mathrm{HCl}=170 \%$ Strychnin.

b) Versuche mit Quecksilberjodid-Jodkaliumlösung.

Zur Anwendung gelangte eine Lösung von $13,5 \mathrm{~g} \mathrm{HgCl}_{2}$ mit $49,8 g \mathrm{KJ} z u \quad 1000 c c$.

10. $10 c c$ Strychninlösung wurden mit $10 c c \mathrm{Hg} \mathrm{J}_{2}$ - KJ lösung vermischt und nach 5 Minuten langer Einwirkung der Niederschlag durch Filtration von der Flüssigkeit getrennt. $12 \mathrm{cc}$ Filtrat $=28,82 \mathrm{cc}$ ${ }_{1}^{1 / 10} \mathrm{~N}-\mathrm{K} \mathrm{OH}$; mithin zur Alkaloidsalzbildung verbraucht: $1,97 \mathrm{cc}=$ $\mathbf{1 6 5} \%$ Strychnin.

11. $10 c c$ Strychninlösung wurden mit $10 c c \mathrm{Hg} \mathrm{J}_{2}$-KJ lösung vermischt, mit Wasser auf $100 \mathrm{cc}$ aufgefüllt; nach 5 Minuten langer Einwirkung: $50 c c$ Filtrat $=23,82 c c \quad 1 / 10 \mathrm{~N}-\mathrm{K} \mathrm{OH}$. Verbraucht: $2,36 c c$ $1 / 10 \mathrm{~N}-\mathrm{HCl}=198 \%$ Strychnin.

12. Analog 11 unter Anwendung von $5 c c \mathrm{HgJ}_{2}$-KJlösung. $50 \mathrm{cc}$ Filtrat : $24,17 c c 1 / 10 \mathrm{~N}-\mathrm{KOH}$. Verbraucht: $1,66 c c 1 / 10 \mathrm{~N}-\mathrm{HCl}=$ $139 \%$ Strychnin.

13. Analog 11, jedoch unter Zufügung von $45 c c^{1 / 10} \mathrm{~N}-\mathrm{K} \mathrm{OH}$ zur sauren Alkaloidsalzlösung vor dem Zusatze der $\mathrm{Hg} J_{2}$-KJ lösung. Die Abscheidung der unlöslichen Alkaloidverbindung vollzog sich ungeheuer langsam; erst nach 30 Minuten langem Stehenlassen konnte ein klares Filtrat erzielt werden. $50 c c$ Filtrat $=1,24 c c \quad 1 / 10 \mathrm{~N}-\mathrm{K}$ OH. Verbraucht: $2,52 c c^{1} / 10 \mathrm{~N}-\mathrm{BCl}=212 \%$ Strychnin.

Der unter dieser Versuchsbedingung erhaltene Niederschlag war leicht zersetzlich. Die Flüssigkeit enthielt in Folge dessen eine geringe Menge freien Jods. ${ }^{1}$ )

14. Analog 11 unter Zusatz von $3 g$ in Wasser gelösten Jodkaliums. Nach 20 Minuten langer Einwirkung $50 \mathrm{cc}$. Filtrat $=23,92 \mathrm{cc}$ $1 / 10 \mathrm{~N}-\mathrm{K} \mathrm{OH}$. Verbraucht: $2,16 c c{ }^{1} / 10 \mathrm{~N}-\mathrm{HCl}=181 \%$ Strychnin.

1) Für die Gesainmtfüssigkeit berechnet sich eine $0,25 \mathrm{cc} 1 / 10 \mathrm{~N}$-Lösung entsprechende Menge freien Jods. 
15. Analog 11 unter Anwendung von $30 \mathrm{cc} \mathrm{HgJ}_{2}-\mathrm{KJ}$ lösung $50 \mathrm{cc}$ Filtrat $=23,8 c c^{1} /{ }_{10} \mathrm{~N}-\mathrm{KOH}$. Verbraucht: $2,4 c c^{1} / 10 \mathrm{~N}-\mathrm{HCl}=\mathbf{2 0 1} \%$ Strychnin.

16. Analog 15, doch wurde die Strychninlösung in die im Ueberschusse angewandte $\mathrm{Hg} \mathrm{J}_{2}$ - $\mathrm{KJ}$ lösung gegossen. $50 \mathrm{cc}$ Filtrat $=24,1 \mathrm{cc}$ $1 / 10 \mathrm{~N}-\mathrm{K} \mathrm{OH}$. Verbraucht: $1,8 c c{ }^{1 / 10} \mathrm{~N}-\mathrm{HCl}=151 \%$ Strychnin.

17. $30 \mathrm{cc}$ Strychninlösung mit $10 \mathrm{cc} \mathrm{Hg} \mathrm{J}_{2}-\mathrm{KJ}$ lösung, analog 10 behandelt. $\quad 25 c c$ Filtrat $=90,93 c c 1 / 10 \mathrm{~N}-\mathrm{K}$ OH. Verbraucht: $4,52 c c$ $1 / 10 \mathrm{~N}-\mathrm{HCl}=126 \%$ Strychnin.

18. Analog 17 , doch nach Fällung mit Wasser auf $100 \mathrm{cc}$ aufgefüllt. Die Flüssigkeit blieb lange Zeit sehr trübe. $25 \mathrm{cc}$ Filtrat $=$ $36,4 c c{ }^{1} /{ }_{10} \mathrm{~N}-\mathrm{K} \mathrm{OH}$. Verbraucht: 4,4 cc $1 /{ }_{10} \mathrm{~N}-\mathrm{HCl}=\mathbf{1 2 3} \%$ Strychnin.

19. $20 \mathrm{cc}$ einer 1 procentigen Strychninlösung mit der gleichen Säuremenge wie oben $\left(=50 c c{ }^{1} / 10 \mathrm{~N}-\mathrm{HCl}\right)$, ergab unter Anwendung von $30 \mathrm{cc} \mathrm{Hg} \mathrm{J}_{2}$ - KJlösung nach 5 Minuten langer Einwirkung ein Filtrat, von welchem $50 c c=21,62 c c 1 / 10 \mathrm{~N}-\mathrm{K} \mathrm{OH}$ entsprachen. Verbrancht: $6,76 c c^{1} / 10 \mathrm{~N}-\mathrm{HCl}=113 \%$ Strychniu.

Diese Beispiele lassen zur Genüge erkennen, dass bei beiden Methoden einerseits der Gehalt an überschüssiger Säure, andererseits der an Jodkalium der Lösungen, endlich auch die Concentration bei der Fällung, einen wesentlichen Einfluss aef das analytische Resultat ausübt. Es kommt dies bei Anwendung von Jod-Jodkalinmlösung in verschärftem Maasse zum Ausdruck. Die Erklärung hierfür ist wohl in der Bildung von Salzen zu finden, die pro Molecül der Alkaloidbase mehrere Molecüle Säure gebunden enthalten, so dass also nicht die Verbindungen (Alkaloid $\mathrm{HJ})_{\mathrm{m}} \mathrm{J}_{\mathrm{n}}$ und (Alkaloid $\left.\mathrm{HJ}\right)_{\mathrm{m}}\left(\mathrm{Hg} J_{2}\right)_{\mathrm{n}}$ entstehen, sondern solche der $\mathrm{Zn}$ sammensetzung

Alkaloid $(\mathrm{HJ})_{\mathrm{m}} \mathrm{J}_{\mathrm{n}}$ und Alkaloid $(\mathrm{HJ})_{\mathrm{m}}\left(\mathrm{HgJ}_{2}\right)_{\mathrm{n}}$

gebildet werden, wobei auch noch Nebenreactionen stattfinden können, auf die an dieser Stelle nicht näher eingegangen werden soll, da dieselben für die Zwecke der vorliegenden Arbeit durchaus nebensächlich sind.

Nun hat zwar Gordin im Archiv der Pharmacie 1901 (239), S. 645 für die Chinaalkaloide angegeben, dass dieselben - wohl im Gegensatze zu seinen im Archiv der Pharmacie 1900, S. 310 publicirten Angaben - als $\mathrm{zw}$ eisäurige Basen zu berechnen sind, entgegen der Zusammensetzung der bis jetzt von ihm untersuchten Basen, welchen die Formel Alkaloid HJ.J zukomme (Archiv der Pharmacie 
1901, S. 646). Ich muss aber die Erzielung gleichmässiger Zahlen auch bei den Chinalkaloiden bezweifeln, indem ich auf die älteren Arbeiten von mir verweise ${ }^{1}$ ), in welchen ich durch analytisch genaux verfolgten Verbrauch an Jodkalium zeigte, dass hier, je nach dem Jodkaliumgehalt der Jodlösung, Gemische von ein- und mehrsäurigen Verbindungen des Chinins entstehen.

Was oben für Strychnin an der Hand analytischer Thatsachen gezeigt wurde, gilt auch für die meisten der anderen Alkaloide. Diesbezügliche Versuche sind überflüssig, da solche mit Bezug auf den Verlauf der quantitativen Umsetzung von Jodkalium der Jodlösung bereits früher von mir durchgeführt wurden, und ich auf das diesbezügliche ausführliche Analysenmaterial verweise ${ }^{2}$ ), andererseits aber auch die mehr oder weniger stark zum Ausdrucke kommende Analogie zum Strychnin in den vorliegenden Fällen logischer Weise vorausgesehen werden kann.

Naheliegend wäre es, direct von den neutralen Alkaloidsalzlösungen, beziehungsweise von in nur wenig überschüssiger Süure gelöstem Alkaloid auszugehen. Es treten dann aber sich ohne Weiteres ergebende Schwieriglkeiten ein. Zunächst ist die Herstellung neutraler oder nahezu neutraler Lösungen sehr schwierig, da man ja die Menge des reinen Pflanzenalkaloids bestimmen will, andererseits zeigen - namentlich bei Anwendung von Quecksilberjodid-Jodkaliumlösung - die Niederschläge alsdanm Zersetzlichkeit (Versuch 13; siehe auch Versuch 25), und endlich und das ist das Wichtigste - sind auch hier die Zahlen durchans keine den einsäurigen Basen entsprechende (siehe Versuch 7, 13, 19, 22, 25). Dazu kommt, dass sich bei einzelnen Alkaloiden mit nur wenig überschüssiger Säure auch nur schwer eine Lösung erzielen lässt. So zeigte die in Versuch 19 benutzte Strychninlösung bereits nach halbstündigem Stehen trotz Anwendung überschüssiger Säure eine starke Abscheidung. Auch der Vorschlag Gor din's, die anzuwendende Säurelösung unbekannten Gehaltes gegen eine Morphinlösung bekannten Gehaltes für die Untersuchung sämmtlicher Alkaloide einzustellen, ist gänzlich verfehlt, wie folgende Versuche zeigen, bei denen Morphin der Zusammensetzug $\mathrm{C}_{17} \mathrm{H}_{19} \mathrm{NO}_{3}$ + aq. in einem dem

1) Diese Zeitschrift $\mathbf{3 5}, 438,435$ und 439.

2) Diese Zeitschrift 35: Für Narcotin 12, 14, 438; für Atropin 13, 14, 15, 17; für Stry chnin 13, 14, 15, 16; für Morphin 439; für Brucin 439 für Coffeün diese Zeitschrift 39, 445. 
Strychnin in oben benutzten Lösungen äquivalenten Verhält$n$ isse $(0,7256+100 c c \mathrm{~N}-\mathrm{H} \mathrm{Cl}$ auf $200 c c)$ zur Anwendung gelangte.

a) Versuchemit Jod-Jodkaliumlösung.

20. $30 \mathrm{cc}$ Morphinlösung, analog Versuch 5: $20 \mathrm{cc}$ Filtrat $=53,04 \mathrm{cc}$ $1 / 10 \mathrm{~N}-\mathrm{K} \mathrm{OH}=116 \%$ Alkaloid, gegenüber $146 \%$ Alkaloid bei Strychnin.

21. $10 c c$ Morphinlösung, analog Versuch $6: 25 c c$ Filtrat $=34,42 c c$ $1 / 10 \mathrm{~N}-\mathrm{KOH}=158 \%$ Alkaloid, gegenüber $268 \%$ Alkaloid bei Strychnin.

22. $30 \mathrm{cc}$ Morphinlösung, analog Versuch 7: Analoge Beobachtung wie dort mit Bezug auf die Abscheidung, doch $50 \mathrm{cc}$ Filtrat $=2,36 \mathrm{cc}$ $1 / 10 \mathrm{~N}-\mathrm{KOH}=155 \%$ Alkaloid, gegenüber $134 \%$ Alkaloid bei Strychnin.

b) Versuche mit Quecksilberjodid-Jodkaliumlösung.

23. $10 \mathrm{cc}$ Morphinlösung, analog Versuch 11: $50 \mathrm{cc}$ Filtrat $=24,12 \mathrm{cc}$ $1 / 10 \mathrm{~N}-\mathrm{KOH}=147 \%$ Alkaloid, doch bleibt trotz Ueberschuss an $\mathrm{Hg} \mathrm{J}_{2}-\mathrm{KJ}$ lösung ein beträchtlicher Theil des Morphins in Lösung.

24. Derselbe Versuch unter Anwendang von $30 \mathrm{cc} \mathrm{Hg} \mathrm{J}_{2}$ - KJ lösung analog 15: $50 \mathrm{cc}$ des fast alkaloidfreien Filtrats $=24,07 c c^{1 / 10} \mathrm{~N}-\mathrm{KOH}$ $=156 \%$ Alkaloid gegenüber $201 \%$ Alkaloid beỉ Strychnin.

25. Derselbe Versuch unter Vermischen der Morphinlösung mit $45 \mathrm{cc} 1 / 10 \mathrm{~N}$-KOH lösung. Nach 10 Minuten: $50 c c$ Filtrat $=1,73 c c$ $1 / 10 \mathrm{~N}-\mathrm{KOH}=129 \%$ Alkaloid, doch auch hier vollständige $\Delta$ bscheidung des Alkaloids unmöglich. Zersetzlichkeit des Niederschlags wie in Versuch 13.

Die Wahl des Morphins ist eine um so unglücklichere, als die Ausfällung dieses Alkaloids mit $\mathrm{Hg} \mathrm{J}_{2}-\mathrm{KJ}$ lösung nur unter Anwendung eines grossen Ueberschusses des Reagens mit einigermaassen befriedigender Vollständigkeit gelingt (siehe Versuch 23-25).

Wenn in einer Alkaloidsalzlösung die Fällbarkeit mit $J$-KJlösung oder mit $\mathrm{HgJ}_{2}$-KJlösung ohne Weiteres ein titrationsfähiges Filtrat erzielen lassen soll, so muss ferner berücksichtigt werden, welcher Art die Reactionsfähigkeit der Verunreinigungen des isolirten Alkaloids sowohl der Säure wie dem Fällungsmittel gegenüber sein wird. In Fällen der pharmaceutischen und der toxikologischen Praxis - für diese speciell ist die Methode von Gordin empfohlen worden - handelt es sich 
hierbei zumeist um die Verunreinigung durch Proteïnstoffe und deren weitere Abbauproducte. Dass auch durch diese eine gewisse Menge der Säure bei der Fällung gebunden wird, ist vorauszusehen. Cholin und Betaïn, die sich beide in vielen pflanzlichen Auszügen vorfinden ${ }^{1}$ ), bei der Bestimmung des wirksamen Alkaloids aber nicht mit in Rechnung gezogen werden dürfen, ergeben ebenfalls unter diesen Fällungsbedingungen Salze, die eine weitere wesentliche Fehlerquelle in der titrimetrischen Bestimmung der wirksamen Alkaloide verursachen.

Die maassanalytische Bestimmung der Pflanzenalkaloide gelingt aber ganz gut durch die directe Titration (Anwendung eines Ueberschusses an Säure und Zurücktitriren des letzteren), sofern man den für das zu titrirende Alkaloid brauchbaren Indicator wählt. Eine diesbezügliche ausführliche Studie ist von mir in dieser Zeitschrift 39, $201 \mathrm{ff}$. erschienen. Meine Versuche sind später im Breslauer chemischen Laboratorium durch E. Springer nachgemacht worden. ${ }^{2}$ ) Springer konnte meinen Beobachtungen nichts Neues hinzufügen und er konnte auch die von mir mit analytischen Daten veröffentlichte Beobachtung der Dissociationsfähigkeit der Alkaloidsalze in wässerigen Lösungen ${ }^{3}$ ) - auch sauren Lösungen - bestätigen. Diese Dissociationsvorgänge können nun eine Rolle spielen, wenn es sich um die quantitative Bestimmung der Alkaloide in pharmacentisch wichtigen Präparaten handelt, bei welcher der titrimetrischen Bestimmung eine Ausschüttelung des Alkaloids voranzugehen hat. Wie sich in solchen Fällen der Einfluss der Dissociationsvorgänge so gut wie gänzlich umgehen lässt, ist aus den analytischen Daten meiner citirten Arbeit zu ersehen. Die im Anschlusse an derartige theoretische Ueberlegungen unternommene Nachprüfung der analytischen Methode des neuen deutschen Arzneibuches ergab unter Benutzung reiner Strychnin- und Brucinlösungen Zahlen, die erkennen lassen, dass auch dem Früfungsmodus des deutschen Arzneibuchs in Folge der Dissociationsfähigkeit der Alkaloidsalze Fehlerquellen anhaften, dass aber dieselben unter den vorgeschriebenen Versuchsbedingungen zu gering sind, um in der pharmaceutischen Praxis unbedingt beachtet werden zu müssen. Unter peinlichster Vermeidung

1) Apotheker-Zeitung 1898, S. 665.

2) Der Alkaloidnachweis. Breslau. Commissionsverlag von W. Koebner 1902 ; siehe auch Apotheker-Zeitung 1902, 225.

\$) Diese Zeitschrift $\mathbf{3 9}, 290$ ff. 
jeglicher Fehlerquelle erhielt ich bei den vom deutschen Arzneibuch vorgeschriebenen Concentrationsverhältnissen einen Verlust an Alkaloid, der 0,3 bis $0,5 c c 1 / 100$ Normal-Lösung für $1 g$ Brechnussextract entsprach.

Königsberg i. Pr., Pharmaceut. - chemisches Laboratorium der Universität.

Die Pigmente der "Radix Anchusa tinctoria" in ibrer Bedeutnng für die alkalimetrische und acidimetrische Analyse. von

\section{A. Gawalowski.}

Diejenigen Forscher, welche sich meines Wissens eingehend mit dem »Alkannaroth $\ll$ befassten, insbesondere Bolley, Pelletier, Wydler und Andere bezeichnen alle das Alkannaroth als den wesentlichen Pigmenttheil der Schlangenwurz. Pelletier ${ }^{1}$ ) gibt diesem Rothpigment den Namen Anchusasäure, weil sein saurer Charakter erwiesen ist.

Bolley und Wydler ${ }^{2}$ ) haben nachgewiesen, dass das Alkannaroth in Gegenwart ron Alkohol und eines, der Wurzel innewohnenden braunen (humösen, harzigen?) Körpers und insbesondere bei Lichteinwirkung in ein grünes Pigment, das »Alkannagrün«, übergeht. Ich fand, dass durch Extraction mit Benzin ein rothes Pigment erhalten wird, welches sich gegen Ammon und Alkalien anders verhält als das nachher aus der Wurzel mittelst Alkohol-Aethers gewonnene zweite rothe Pigment. Daher schlage ich vor, ersterem den Namen Anchusasäure oder Anchusaroth, letzterem aber den Namen Alkannasäure oder Alkannaroth zu geben. Dem wahrscheinlich durch Aldehydbildung (Wasserstoffaustritt aus dem Alkohol, Sauerstoffaustritt aus dem Pigment, Wasserbildung and wahrscheinlich Wasserstoffeintritt in das Molecül des Alkannarothes) entstehenden Alkannagrün mag der Name bleiben.

Alkannasäure (Alkannaroth) mit der wahrscheinlichen empirischen Formel $\mathrm{C}_{15} \mathrm{H}_{14} \mathrm{O}_{4}{ }^{3}$ ) wird durch Ammon aus roth in ultra-violettblau, dureh Alkalilauge in indigo-blau verwandelt, dagegen wird Anchusasäure (Anchusaroth) mit der wahrscheinlichen empirischen Formel $\mathrm{C}_{30} \mathrm{H}_{39} \mathrm{O}_{7}$ durch

1) Annalen d. Physik u. Chemie 51.

2) Annalen d. Chemie $u$. Pharmacie 62.

3) Oder: $2\left(\mathrm{C}_{15} \mathrm{H}_{14} \mathrm{O}_{4}\right)=\mathrm{C}_{30} \mathrm{H}_{28} \mathrm{O}_{8}$ im Vergleich zu der empirischen Formel der Anchusasäure $\mathrm{C}_{30} \mathrm{H}_{39} \mathrm{O}_{7}$. 